\title{
Order Twins in CuAu Films Formed Epitaxially by Vacuum Deposition*
}

\author{
By Saiyu Maruyama** and Katsumi Yamamoto**
}

\begin{abstract}
By means of electron diffraction and lattice imaging microscopy, new modes of order twin were found in films of the composition around $\mathrm{Cu}-50$ at. $\% \mathrm{Au}$ made epitaxially by successive deposition in vacuum, homogenized and slowly cooled from the disordered state to room temperature. Order twins (101) change the form and the size depending on the composition or the tetragonality, $c / a$. In $\mathrm{Cu}-40$ or 60 at. $\% \mathrm{Au}$ films, in which the tetragonality is closer to unity, the twinned layers are several tens nanometer in breadth, and the twin boundaries are distinct and parallel to the (101) twin planes which are normal to the film plane, while in $\mathrm{Cu}-50$ at. \% Au films, in which $c / a$ reaches 0.927 , the twinned regions are as narrow as several atomic layers, and the twin boundaries do not always coincide with the (101) twin planes but become obscure. They are often the (100) plane which is parallel to the film surface. In the last case, the misfit and overgrowth between two sheets produce a large measurable lattice stretch, which is characteristic of thin films.
\end{abstract}

(Received March 11, 1983)

Keyzuords: order twins, copper-gold thin films, extended Pashley's method, lattice imaging, stress accommodation

\section{Introduction}

The alloy $\mathrm{CuAu}$, which has a $f c c$ disordered structure above $683 \mathrm{~K}$, becomes ordered below this temperature and forms two types of superlattice. Below $653 \mathrm{~K}$, it has a tetragonal structure called $\mathrm{CuAuI}$, and has the alternate (002) planes of all gold and all copper atoms, giving an axial ratio $c / a$ of 0.927 . Between 653 and $683 \mathrm{~K}$, an orthorhombic structure, CuAuII appears, in which gold and copper atoms in $\mathrm{CuAuI}$ alternate their positions at every fifth cell along one of $a$-axis, across the (100) or (010) antiphase boundaries.

Twins formed during ordering from the disordered state and having (101) twin plane are called order twins. Due to the tetragonal distortion the lattice produces a tensile stress in the $c$-direction, and compressive stresses in the other $a$-directions, so that the $c$-axis alternates in three directions from domain to domain in order to accommodate these stresses. The alternation of the $c$-axis is achieved by forming the (101) twin configuration. This

* Paper partly presented at the 10th Int. Congress on Electron Microscopy, Hamburg, 1982.

* Department of Natural Science, Osaka Women's University, Daisen-cho, Sakai, Osaka 590, Japan. is because only this plane scarcely changes the area during ordering (less than $1 \%$ ), and the ordering may occur first along this plane in the form of (101) twins.

When the order twins are formed upon heating from the quenched disordered state, they have a form of microtwins with several tens nanometer in thickness, or a form of coarse twins up to 1 micron depending on the heat treatment ${ }^{(1)-(3)}$. When they are formed upon cooling from the disordered state, which were exclusively observed in thin films prepared by vacuum deposition ${ }^{(2)(4)}$, or by sputtering $^{(5)}$, the twinning proceeds very rapidly ${ }^{(4)}$, and gives the $c$-axis distribution in three directions in a fine scale. They are the same in nature as accommodation twins formed during martensite transformation. In $\mathrm{Cu}-40$ at. \% Au films, especially, the $c$-axis alternates solely in the film plane and the order twins provide a cross stripes pattern ${ }^{(6)}$.

\section{Experimental}

For the purpose of lattice imaging of alloy films, Pashley's method for gold film preparation $^{(7)}$ was extended to prepare copper-gold films as described in the following. A gold film from 10 to $20 \mathrm{~nm}$ in thickness, almost hole free, was put on a flat surface of a rock salt, 
which had been cloven and polished using water and alcohol in order to remove cleavage ledges. This was done in pure alcohol. The deposition of copper was done on this gold film at $573 \mathrm{~K}$, and then homogenized and ordered at the same temperature for $3 \mathrm{~h}$. In some cases, the film was detached from the substrate, mounted on a molybdenum grid and heat-treated at higher temperatures.

The composition was determined by measuring the lattice constants of the disordered state by electron diffraction. The composition agrees with the expected value within 1 or 2 per cent, proving this extended Pashley's method to be very usuful for the preparation of epitaxial alloy films thin enough for lattice imaging.

Images and diffraction patterns were taken with the use of a side-entry goniometer attached in JEM 100CX. The objective pole piece was specially improved for high resolution imaging.

\section{Results and Discussions}

In the $\mathrm{CuAu}$ films, which were disordered at $723 \mathrm{~K}$ and cooled slowly to the ordering temperature, many types of order twins were found. Figure 1 shows the (101) ordinary order twins in $\mathrm{Cu}-60$ at. $\% \mathrm{Au}$, where the lattice fringes corresponding to the (001) spacing of $0.380 \mathrm{~nm}$ are seen. In this case, the $c$-axis has a tendency to lie in the film plane, and alternates its direction from domain to domain forming the (101) type order twins. The thickness of the twin is ten nanometer on the average, which is thinner than the microtwins already found ${ }^{(1)-(6)}$, and does not vary by further annealing. In Fig. 2 for a $\mathrm{Cu}-50$ at. $\% \mathrm{Au}$ film, two kinds of 001 reflection are excited, and the lattice fringes with spacing $0.368 \mathrm{~nm}$ are seen. The (101) twin relation holds, but since the domain thickness is of the order of about ten (001) planes, twin boundaries are

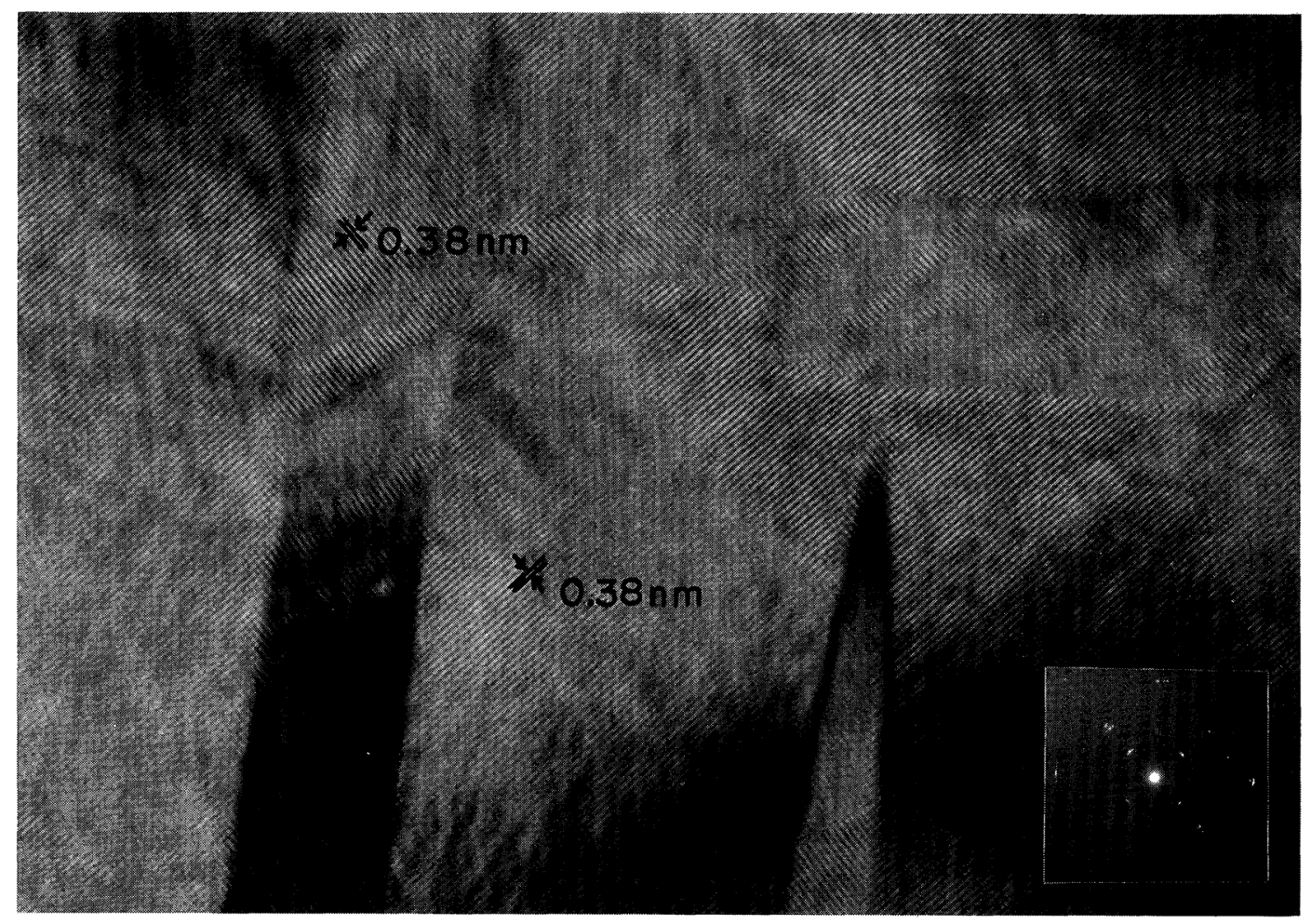

Fig. 1 (101) order twins in a $\mathrm{Cu}-60$ at. $\% \mathrm{Au}$ film slowly cooled from the disordered state. The (001) lattice fringes give the spacing of $0.380 \mathrm{~nm}$. The (101) twin boundaries are normal to the film plane. Average twin thickness is $10 \mathrm{~nm}$. 


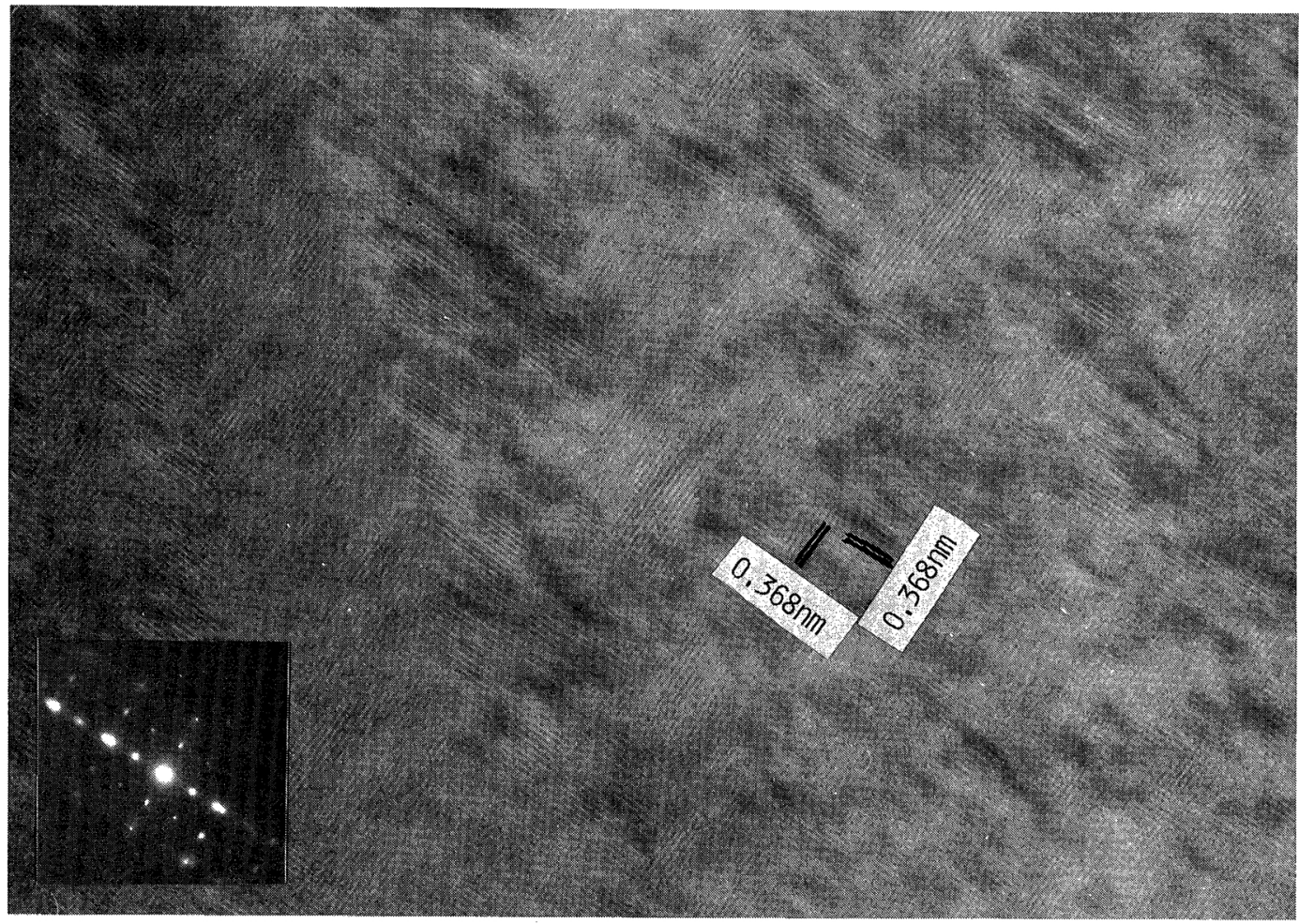

Fig. 2 Order twins in a $\mathrm{Cu}-50$ at. $\% \mathrm{Au}$ film. Two kinds of 001 reflection are excited, giving the lattice fringes $0.368 \mathrm{~nm}$ in spacing. They are (101) order twins, but the domains are of the order of ten (001) layers, and do not show any distinct boundaries.

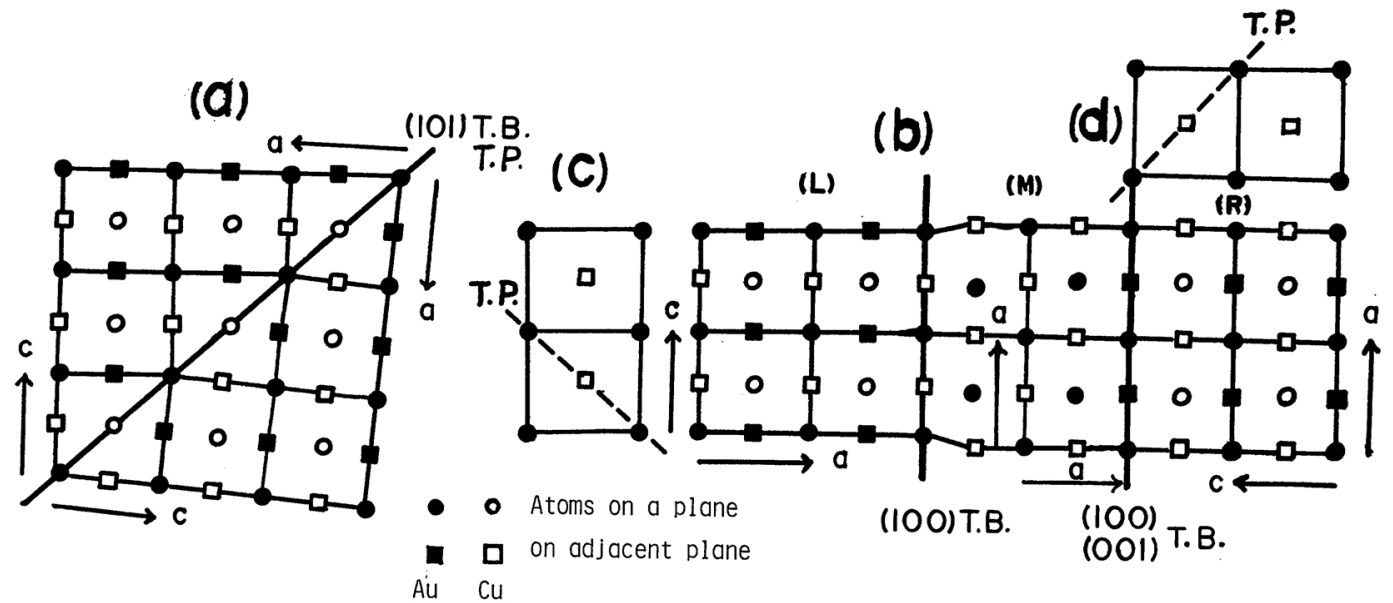

Fig. 3 Order twin configurations in CuAuI. They have (101) twin plane in common (denoted by T.P.), but have different twin boundaries (denoted by T.B.); (101) in (a), and (100) or (001) in (b). Here atoms on a plane parallel to the film surface are represented by circles and those on the adjacent plane are by squares. Gold and copper atoms are distinguished by solid and open circles or sqares. (a) and (b): Views along the normal of the film. (c) and (d): Side views. 


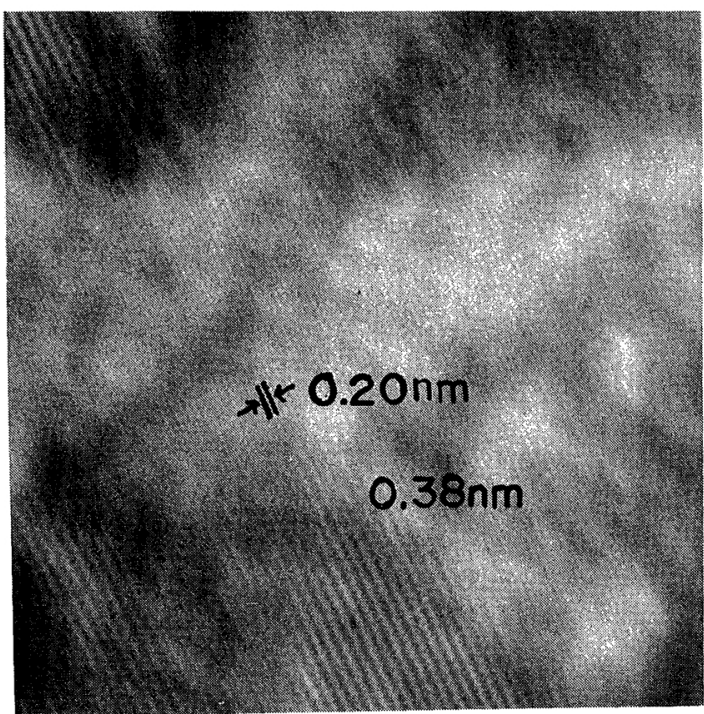

not clear. The difference in the domain thickness and that in the manner of distribution of the order twins are probably due to the difference in the tetragonality $c / a$, which is 0.953 in $\mathrm{Cu}-40$ or 60 at. $\% \mathrm{Au}$, and 0.927 in $\mathrm{Cu}-50$ at. $\% \mathrm{Au}$. As the former is less distorted by ordering, stress accommodation could be done by a large twin formation, in contrast to the latter submitting substantially a large distortion and consequently the stress accommodation in nano scale.

Besides the above ordinary order twins, new

Fig. 4 New modes of order twin in a $\mathrm{Cu}-60$ at. $\% \mathrm{Au}$ film with $c$-axes parallel and normal to the film surface. Lattice fringes (001) and (200) are observed with spacings of 0.38 and $0.20 \mathrm{~nm}$, respectively. Twin boundaries are obscure.

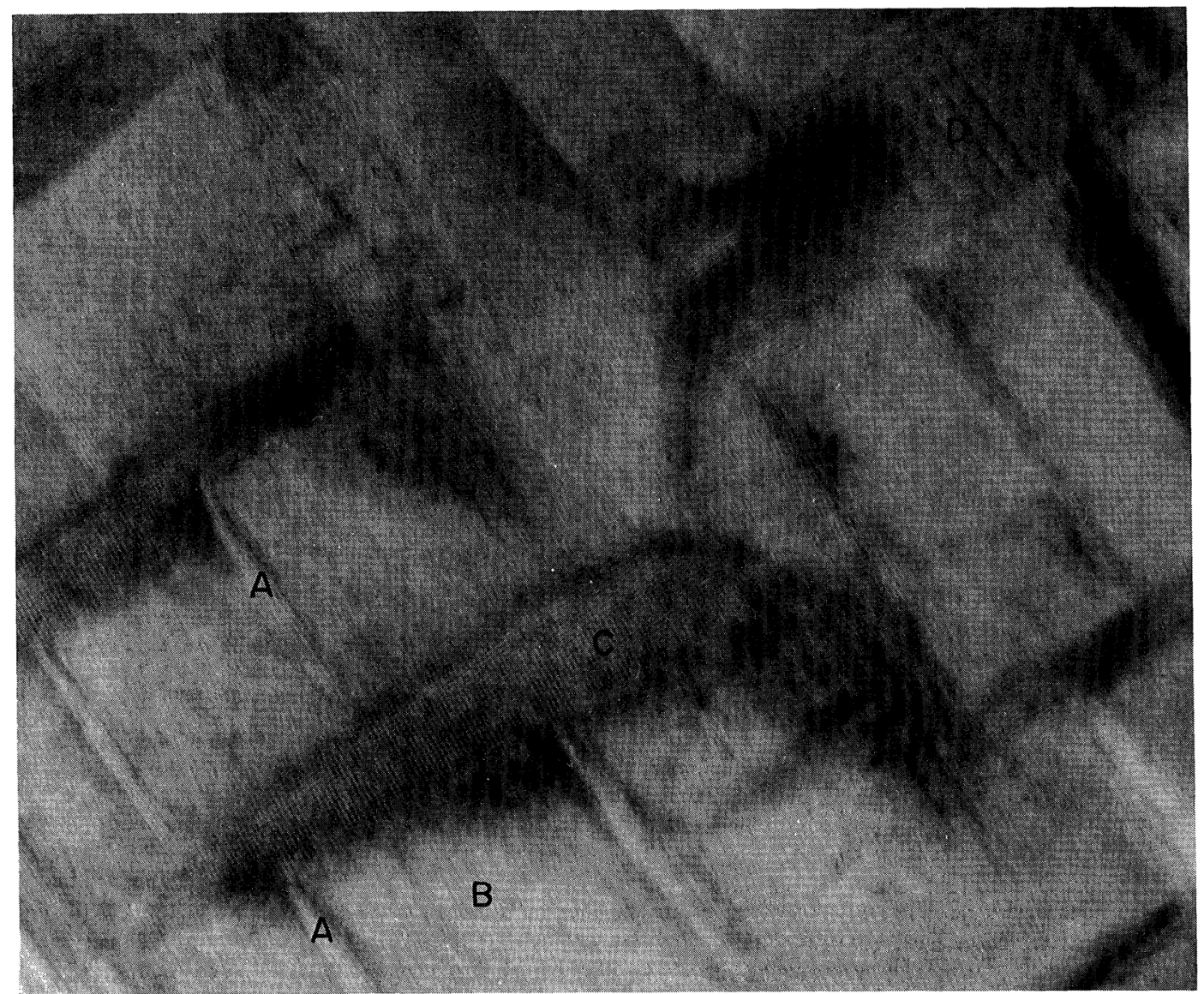

Fig. $5 \mathrm{Cu}-50$ at. \%Au film. A, B and C are the regions respectively with $c$-axes parallel, normal to the film, and overlapping each other. (001) lattice fringes are seen in A and C. A and B have (001) or (100) twin boundaries normal to the film surface, while in C, overlapping two sheets have the twin boundary plane (100) parallel to the film surface. Antiphase boundaries (100) are seen in D. 


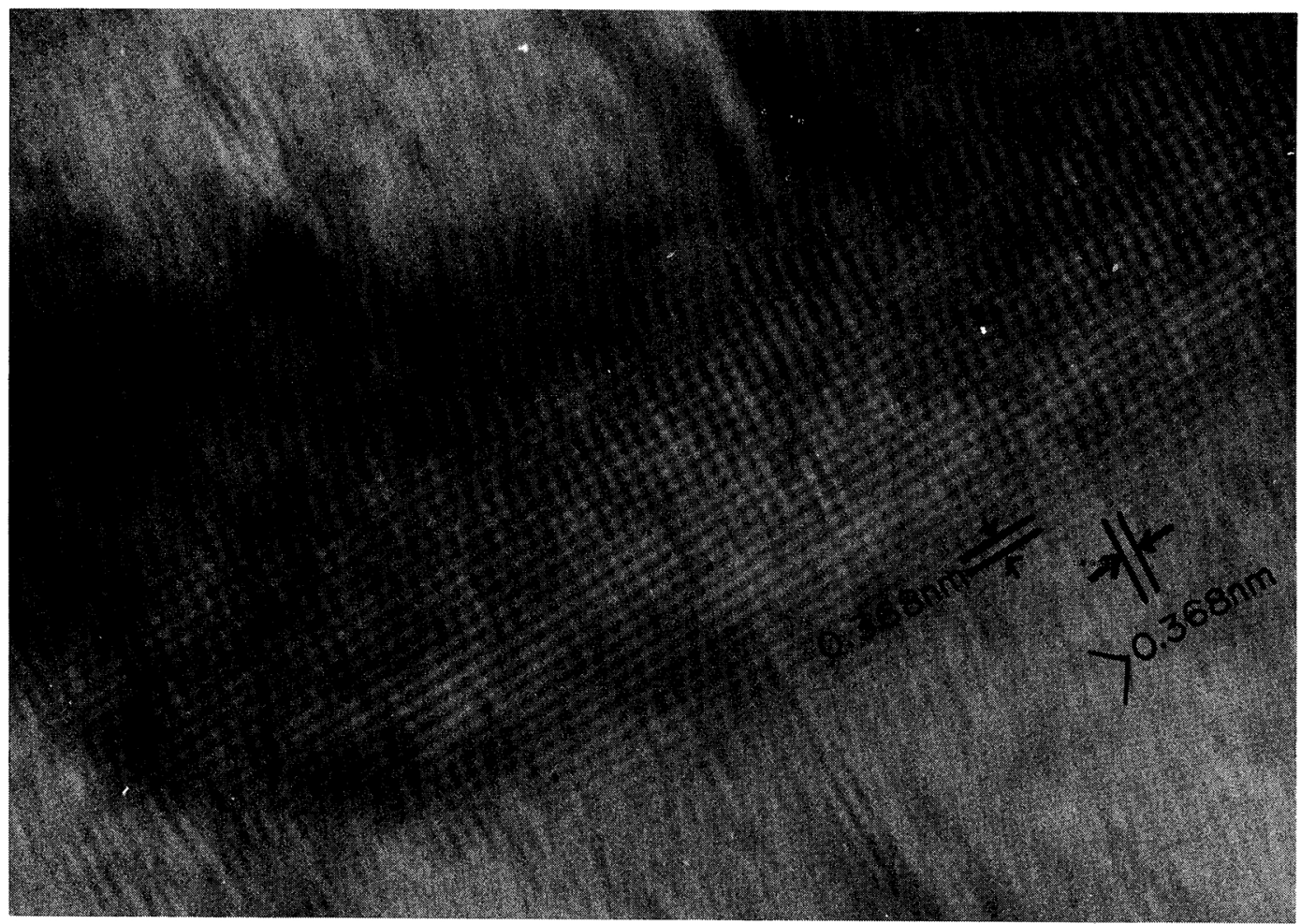

Fig. 6 Enlarged view of the part $\mathbf{C}$ in Fig. 5. Lattice spacing (001) is larger in the longer direction of the domain. Two overlapping (001) planes make an angle of $85^{\circ}$ as a result of (101) twinning.

modes of order twins were found. They are always in the (101) twin relation, but the twin boundary does not coincide with the twin plane (101); it is either the other planes such as (100), or obscure. They are illustrated in Fig. $3(b)$, together with the ordinary order twins in Fig. 3(a). In Fig. 4, which shows the (101) order twin in the $\mathrm{Cu}-60$ at. $\% \mathrm{Au}$ film, the lower part gives the (001) lattice fringes and the $c$-axis lies in the film plane. But in the middle part the lattice fringes have a spacing a little wider than that of (002), and the contrast of the lattice fringes is homogeneous in the whole area. They are identified as the (200) lattice fringes. Therefore, the two domains have the $c$-axes nearly perpendicular to each other and are in the (101) twin relation corresponding to the (L) and (M) domains in Fig. 3(b), except that the twin boundary is not planar. Figure 5 shows the order twins in the $\mathrm{Cu}-50$ at. $\% \mathrm{Au}$ film. $\mathrm{A}, \mathrm{B}$ and $\mathrm{C}$ are the regions respectively with the $c$-axes being parallel, normal to the film surface and overlapping each other. The (001) lattice fringes are seen in A and C. A and $B$ correspond to $(R)$ and $(M)$ domains in Fig. 3(b) with the (100) plane normal to the film surface as the twin boundary, while in $C$, overlapping two parallel sheets have their $c$-axes lying in the film and nearly perpendicular to each other, as (L) and (R) in Fig. 3(b). They are in the (101) twin relation, but the twin boundary is (100), which is parallel to the film surface. Due to the possible overgrowth and misfit between two domains across the twin boundary, the part $\mathrm{C}$ is heavily distorted, and the spacing of (001) is quite larger along the longer direction of the domain, in the region free from extra plane, compared with the other direction, as is clearly seen in the enlarged view of the part C, in Fig. 6. Here, the two overlapping (001) planes make an angle of $85^{\circ}$ on the average, which is the deviation realized in the stoichiometric $\mathrm{CuAu}$, by (101) twinning. 


\section{REFERENCES}

(1) M. Hirabayashi and S. Weissmann: Acta Met., 10 (1962), 25.

(2) A. M. Hunt and D. W. Pashley: Metallic Solid Solution, Ed. by J. Friedel and A. Guinier, W. A. Benjamin, Inc., 1963, New York, pL-1.

(3) D. W. Pashley, J. L. Robertson and M. J. Stowell:
Phil. Mag., 19 (1969), 83.

(4) S. Maruyama: Bull. Osaka Women's Univ., 1 (1964), 15 (in Japanese).

(5) H. C. Tong and C. M. Wayman: Acta Met., 21 (1973), 1381.

(6) S. Yamaguchi: Japan. J. Appl. Phys., 5 (1966), 496.

(7) D. W. Pashley: Phil. Mag., 4 (1959), 324.

\section{Postscript by the Editor}

Atomic structure is no longer an idealistic picture to symbolize the understanding of materials science. It is an experimental tool to help advance materials engineering. It is to be noted that atom resolution electron microscopy has attracted the attention of many materials scientists in Japan. The present special issue is a first edition of Trans. JIM which reflects special high interests among Japanese metallurgists in the future role of atom imaging electron microscopy for solving long standing structural problems of atomic scale.

Both metallurgists and materials scientists have responded favorably to the present undertaking. In fact all of the researchers who had received individual calls from the editor sent papers for publication in the special edition. The number of the articles went up as many as twenty-one including contributed papers, which gave substantial loads to editing.

I would like to take this opportunity to thank the authors, the editorial committee, and especially Prof. H. Hashimoto of Osaka Univ., Prof. M. Hirabayashi of Tohoku Univ. and Prof. S. Nagakura of Tokyo Institute of Technology for their active cooperation as co-editors.

Yoichi Ishida

Prof. of The University of Tokyo 